\title{
FACTORES DE RIESGO ASOCIADOS A INFECCIÓN DEL SITIO OPERATORIO POST CESÁREA DEL HOSPITAL NACIONAL EDGARDO REBAGLIATI - 2017.
}

\author{
RISK FACTORS ASSOCIATED WITH THE INFECTION OF THE OPERATING SITE POST \\ CESÁREA OF THE NATIONAL HOSPITAL EDGARDO REBAGLIATI - 2017.
}

Díaz-Quispe Rommy ${ }^{1, a}$

1. Dirección Regional de Salud de Ica, Ica, Perú

a. Médico cirujano.

\section{Correspondencia:}

Díaz Quispe Rommy Jackelin. Domicilio: Av. Artemio Molina N¹175 Pueblo Nuevo - Chincha - Ica.

Teléfono: +51931264727

Email: rommydq@gmail.com

Contribuciones De Autoría: DQR: Contribuciones en la concepción y diseño del manuscrito, recolección, análisis e interpretación de los datos; redacción y revisión crítica del contenido del manuscrito y la aprobación final del artículo.

Conflicto De Intereses: No declarados.

Financiamiento: Autofinanciado.

\section{Como Citar}

Como citar: Díaz-Quispe Rommi J. Factores de riesgo asociados a infección del sitio operatorio post cesárea del Hospital Nacional Edgardo Rebagliati - 2017. Rev méd panacea 2019;8(1): 26-30

Recibido: 03 - 03 - 2019

Aceptado: 20 - 04 - 2019

Publicado: 23 - 04 - 2019

\section{RESUMEN}

Objetivo: Identificar los factores de riesgo asociados a infección del sitio operatorio post cesárea del Hospital Nacional Edgardo Rebagliati durante el año 2017. Materiales y métodos: Estudio observacional, transversal, analítico y retrospectivo con diseño de casos y controles. Se realiza la investigación con 83 casos de post cesareadas con infección del sitio operatorio comparado con 166 controles de post cesareadas sin infección del sitio operatorio. Resultados: En el año 2017, se realizaron 4131 cesáreas, representando el $65 \%$ del total de partos, de las cuales $2.69 \%$ (111) presentaron infección de sitio operatoria. Se encontró como factor de riesgo a la obesidad $(p=0.00)$ OR: 5.5 IC 95\% [2.3 - 13.2]. Conclusiones: La obesidad es un factor de riesgo para desarrollar infección de sitio operatorio post cesárea. No se halló asociación significativa para la anemia, la ruptura prematura de membranas, el control prenatal insuficiente ni al tiempo quirúrgico mayor de 60 minutos.

Palabras clave: Factores de riesgo, cesárea, Infección de herida operatoria, obesidad.

\section{ABSTRACT}

Objective: To identify the risk factors associated with infection of the post-cesarean operative site of the Edgardo Rebagliati National Hospital during the year 2017. Materials and methods: Observational, transversal, analytical and retrospective study with case and control design. The investigation was carried out with 83 cases post-cesarean with infection of the operative site compared with 166 controls of post-cesareans without infection of the operative site. Results: In the year 2017, 4131 cesarean sections were performed, representing $65 \%$ of the total deliveries, of which $2.69 \%$ (111) presented operative site infection. Obesity was found to be a risk factor $(p=0.00)$ OR: $5.595 \%$ IC [2.3 - 13.2]. Conclusions: Obesity is a risk factor for developing post-cesarean site infection. No significant association was found for anemia, premature rupture of membranes, insufficient prenatal control or surgical time greater than 60 minutes.

Keywords: Risk factors, caesarean section, surgical wound infection, obesity. 


\section{INTRODUCCIÓN}

En los últimos años, tanto a nivel nacional como internacional y en concordancia con nuestra realidad nacional, se han planteado diversos problemas sanitarios con prioridad para la investigación, dentro de ello se encuentra la salud materna, perinatal y neonatal, siendo tema de este estudio. (1)

Si bien es cierto, actualmente se fomenta, evitar recurrir a la cesárea, a menos que sea necesario, el incremento en las estadísticas es alarmante tanta a nivel nacional como internacional, y como toda cirugía, ésta podría traer complicaciones. (2)

Durante 30 años, los profesionales de la salud de todo el mundo han considerado que la tasa ideal de cesárea debe oscilar entre el $10 \%$ y el $15 \%$, basados en una reunión de expertos organizada por la OMS en 1985. Sin embargo, en los últimos años estas tasas van en aumento, conllevando al aumento de complicaciones. En las Américas, cuatro de cada diez partos son por cesárea, superando el ideal de uno de cada diez considerado por profesionales de la salud de todo el mundo. (2)

Dentro de una de las principales complicaciones, tenemos a la infección de herida operatoria. A pesar del avance de la asepsia y antisepsia, la profilaxis antibiótica, entre otros, la infección de herida operatoria en post cesareadas sigue siendo un problema en nuestro país, la cual trae repercusión a la madre, al neonato y a la salud pública. (3)

En el Perú hasta el 2015, el 35.4\% de partos son por cesárea (4). En el mismo año, en un estudio observacional en Lima-Perú sobre la frecuencia de cesáreas injustificadas, Flores encontró una frecuencia de $35,5 \%(5)$

Según la OPS, la infección de sitio quirúrgico se presenta desde el 1,46 \% al $10 \%$ de las cesáreas, según el país de que se trate (6). La incidencia de infección de herida operatoria tras la cesárea varía entre $2.8 \%$ a $10 \%$, en el Instituto Materno Perinatal- Perú (INMP) para el año 2017 fue de $2.44 \%$ en post cesareadas. (7)

Existen múltiples factores para desarrollar infección de herida operatoria en post cesareadas, dentro los cuales existen factores modificables, de tal manera que, al corregirlos de manera óptima y oportuna, se puede evitar la serie de consecuencias que pueda traer esta, es por ellos la motivación de realizar la siguiente investigación.

\section{MATERIALES Y MÉTODOS}

Es un estudio observacional, transversal, analítico y retrospectivo con diseño de casos y controles. Se realiza la investigación con 83 casos de post cesareadas con infección del sitio operatorio comparado con 166 controles de post cesareadas sin infección del sitio operatorio, en el Hospital Nacional Edgardo Rebagliati en el año 2017.

Los datos se recolectaron de las historias clínicas, mediante una ficha de recolección de datos, la cual fue tomada de la autora Richter (10).

Se elaboró tablas de contingencia de $2 \times 2$, en base a la información recolectada y fueron transferidas al programa SPSS 23.0 en donde se realizó el análisis de los siguientes factores de riesgo:

- Anemia (Hemoglobina < $11 \mathrm{mg} / \mathrm{dl}$ )

- Ruptura prematura de membranas

- Obesidad (IMC <30)

- Controles Prenatales Insuficientes (< de 6 (PN)

- Tiempo quirúrgico prolongado (> 60 minutos)

Se analizó la asociación que existe entre las variables mediante la prueba de Chi cuadrado y la magnitud de la asociación entre los factores de interés y el evento resultado mediante el OR con un intervalo de confianza al $95 \%$.

\section{RESULTADOS}

Tabla 1: Número de infección del sitio operatorio post cesárea en el Hospital Nacional Edgardo Rebagliati, 2017.

\begin{tabular}{ccc}
$\begin{array}{c}\text { PARTOS } \\
\text { ATENDIDOS }\end{array}$ & CESÁREAS & ISO \\
6325 & 4131 & 111 \\
& $65 \%$ & $2.69 \%$ \\
\hline
\end{tabular}

En el año 2017, en el Hospital Nacional Edgardo Rebagliati se atendieron en total 6325 partos, de los cuales 4131 fueron sometidos a cesárea, representando el 65\%. De ellos 111 mujeres desarrollaron infección del sitio operatorio post cesárea, representando el $2.9 \%$ de las post - cesareadas. En el presente trabajo, las edades estuvieron comprendidas entre 16 y 54 años, con un promedio de edad de 32.16 años. 
Tabla 2: Factores de riesgo asociados a infección del sitio operatorio post- cesárea en el Hospital Nacional Edgardo Rebagliati, 2017.

\begin{tabular}{cccccccc} 
& \multicolumn{2}{c}{ CASOS } & \multicolumn{2}{c}{ CONTROLES } & P & OR (IC 95\%)
\end{tabular}

Anemia

$\begin{array}{lcccccc}\text { - Sí } & 6 & 7.20 \% & 27 & 16.30 \% & 0.047 & 0.4 \\ \text { - No } & 77 & 92.80 \% & 139 & 83.70 \% & & (0.16-1.01)\end{array}$

Ruptura Prematura de Membranas

$\begin{array}{lcccccc}\text { - Sí } & 21 & 25.30 \% & 33 & 19.90 \% & 0.32 & 1.4 \\ \text { - No } & 62 & 74.70 \% & 133 & 80.10 \% & & (0.73-2.55)\end{array}$

Obesidad

- Sí (IMC $\left.\geq 30 \mathrm{Kg} / \mathrm{m}^{2}\right)$

- No (IMC $<30 \mathrm{Kg} / \mathrm{m}^{2}$ )

Controles Prenatales Insuficientes

- Sí (<6)

- No ( $\geq 6)$

31

$37.30 \%$

50

$30.10 \%$

0.25

52

$62.70 \%$

116

$69.90 \%$

Tiempo quirúrgico prolongado

- Sí (>60 min.)

- No ( $\leq 60 \mathrm{~min}$.)
$80 \quad 96.40 \% \quad 155 \quad 93.40 \%$

$3 \quad 3.60 \% \quad 11 \quad 6.60 \%$
1.4

$(0.79-2.40)$

5.5

$(2.3-13.2)$

0

)

Fuente: Historias Clínicas del Hospital Nacional Edgardo Rebagliati.

Al realizar el análisis, se encontró como factor de riesgo a la obesidad $(p=0.00)$ OR: 5.5 IC 95\% [2.3-13.2]. No se identificó asociación a la anemia, la ruptura prematura de membranas, el control prenatal insuficiente ni al tiempo quirúrgico mayor de 60 minutos.

\section{DISCUSIÓN}

El presente trabajo de investigación, sobre factores de riesgo asociados a infección del sitio operatorio post cesárea, en el Hospital Nacional Edgardo Rebagliati 2017; se encontró que, del total de partos atendidos, el $65 \%$ fue parto por cesárea y dentro de éstas 111 se complicaron con infección del sitio operatorio, representando un 2.9\% del total de las cesáreas. La OMS, recomienda que la tasa ideal de cesárea debe oscilar entre 10 al 15\% (1). Según la OPS, la tasa de infección de sitio quirúrgico se presenta desde el 1,46\% al $10 \%$ de las cesáreas, según el país de que se trate (6). En el año 2017, en el Instituto Materno Perinatal- Perú, la tasa de ISO - post cesárea fue de $2.44 \%$ (7).

Con respecto a los factores de riesgo asociados a la infección de sitio operatorio, que es en lo que estuvo centrada esta investigación, se analizaron los siguientes factores, destacando en orden de frecuencia: La duración del procedimiento quirúrgico mayor a 60 minutos en un $96.4 \%$, los controles prenatales insuficientes en $37.3 \%$, la ruptura prematura de membranas en $25.3 \%$, la obesidad en $21.7 \%$ y la anemia en $7.2 \%$.

Al buscar asociación, se encontró diferencia estadísticamente significativa, al analizar la obesidad como factor de riesgo, encontrándose que ésta favorece hasta 5.5 para desarrollar ISO post cesárea. un $\mathrm{p}=0.00$, IC 95\% [2.3 - 13.2].

Sin embargo, se determinó que la anemia, la ruptura prematura de membranas, los controles prenatales insuficientes y la duración del procedimiento quirúrgico mayor a 60 minutos; no muestran diferencia estadísticamente significativa, no siendo considerados como factor de riesgo en el presente estudio.

Respecto a la obesidad, de la cuál en nuestra investigación encontramos asociación con un $p=0.00$ y 
un $\mathrm{OR}=5.5$. Al respecto Dotters (8) en EE.UU., también refiere que un IMC elevado condiciona al desarrollo de infección de la herida post cesárea $(p=0.04)$. A la vez Salazar (9) en Cajamarca, determinó un OR 5.44 (IC 2.0814.23) $p=0.0003$, para la obesidad. Sin embargo, Richter (10) en su estudio en Lima, no halló asociación significativa entre la obesidad y la ISO post cesárea.

En cuanto a la anemia, no encontramos asociación $p=0.047$ IC $(0.16-1.01)$, difiriendo de Loredo (11) quien concluyó que la anemia durante el tercer trimestre de la gestación sí es factor de riesgo significativo para desarrollar ISO en pacientes post cesareadas con un OR $=6.53$. De igual manera Mamani (12) también encontró asociación con $[\mathrm{OR}=4,39 ;(p<0.05)]$. Sin embargo, Ritcher (10), en su estudio tampoco encontró asociación entre la anemia y la ISO post cesárea.

Respecto a la ruptura prematura de membranas,

\section{REFERENCIAS BIBLIOGRÁFICAS}

1. Instituto nacional de salud (INS). Aprobación y publicación de Prioridades Nacionales de Investigación en Salud en el Perú 2016 -2021. [Internet]. Lima (Perú); 2016 [citado 20 de noviembre del 2018]. Disponible en: http://www.sld.cu/galerias/pdf/sitios/histologia/no rmas-vancouver-buma-2013-guia-breve.pdf

2. Organización Mundial de la Salud (OMS). Declaración de la OMS sobre tasas de cesárea. [Internet]. Ginebra: Departamento de Salud Reproductiva e Investigación de la OMS; 2015 [citado el 3 de diciembre del 2018]. Disponible en: https://apps.who.int/iris/bitstream/handle/10665/1 61444/WHO_RHR_15.02_spa.pdf;jsessionid $=3$ C667 68648E51A1F101CEF409B433A09? sequence $=1$

3. Frias $C N$, Begué $D N$, Martí RL, Leyva FN, Méndez LL. Infección del sitio quirúrgico poscesárea. MEDISAN [Internet]. Cuba; 2016. [citado 20 de noviembre del 2018]; 20( 5 ): 596-603. Disponible en: http://scielo.sld.cu/scielo.php?script=sci_arttext\&pi $d=$ S1029-30192016000500002\&lng=es.

4. Ministerio de Salud (MINSA). Boletín estadístico de nacimientos Perú: 2015. Lima (Perú): Oficina general de tecnologías de la información; 2016. [citado 20 de noviembre del 2018].

5. Flores A. Frecuencia de cesáreas injustificadas en el servicio de ginecología y obstetricia del Hospital de Ventanilla durante el año 2015. Lima (Perú). Facultad de Medicina Humana. Universidad Ricardo Palma; 2017. [Citado el 20 de noviembre del 2018]. Disponible en:

http://cybertesis.urp.edu.pe/bitstream/urp/973/1/A ndreaFlores_2017_pdf.pdf tampoco encontramos asociación significativa ( $p=0.32)$. No estando en concordancia con Salazar (9) quien en su estudio determinó asociación con un $\mathrm{OR}=4.53, \mathrm{p}=0.04$. Al igual Miranda (13) en Guatemala halló que existe cinco veces más riesgo de padecer infección de herida si la gestante presenta las membranas rotas mayor de 12 horas.

Tampoco encontramos asociación, al analizar los controles prenatales insuficientes ni la duración del procedimiento quirúrgico mayor a 60 minutos. Richter (10) en su estudio determinó que el tiempo quirúrgico prolongado es factor de riesgo para adquirir una infección de herida operatoria luego de una cesárea, con un OR 9.533 (IC 4.196 - 21.658). Pero, difiere de Salazar (9), quien al igual que este estudio, no halló asociación significativa entre el tiempo quirúrgico prolongado y la ISO post cesárea.

6. Organización Panamericana de la Salud (OPS). Vigilancia epidemiológica de las infecciones asociadas a la atención de la salud en el puerperio, modulo V. [Internet]. Washington, D.C. (EE. UU.): Departamento de Enfermedades Transmisibles y Análisis de Salud; 2018 [citado el 3 de diciembre del 2018]. Disponible en:

https://www.paho.org/hq/dmdocuments/2014/201 4-cha-vigilancia-epi-infecciones-modulo-V.pdf

7. Instituto Nacional Materno Perinatal (INMP). Boletín epidemiológico, consolidado 2017. Lima (Perú): Oficina de epidemiología y salud ambiental del INMP; 2017. [Citado el 3 de diciembre del 2018]. Disponible en: https://www.inmp.gob.pe/institucional/boletin-epid emiologico/1421335605? pagina $=1$

8. Dotters - Katz SK, Feldman, Puechel A. Factores de riesgo para la infección postoperatoria de la herida en el contexto de corioamnionitis y parto por cesárea. J Matern Fetal Neonatal Med. [Internet]. 2016 [Citado 15 de octubre del 2018]; 29 (10): 1541-5. Disponible en:

https://www.ncbi.nlm.nih.gov/pubmed/26135793

9. Salazar Guerrero O. Factores de riesgo de infección de sitio quirúrgico en pacientes sometidas a cesárea en el departamento de gíneco-obstetricia del Hospital Regional Docente de Cajamarca 20132014. [Tesis para optar el grado de Médico Cirujano]. Perú, repositorio de la UNC. Universidad Nacional de Cajamarca; 2015.

10. Richter Soto L. Factores de riesgo asociados a infección de herida operatoria post cesárea en el Hospital Vitarte, periodo 2013 a junio 2015. 


\section{REFERENCIAS BIBLIOGRÁFICAS}

[Tesis para obtener el grado de médico cirujano]. Lima (Perú). Universidad Ricardo Palma; 2016.

11. Loredo Reyes V. Anemia del tercer trimestre de la gestación factor de riesgo para infección de sitio operatorio en puérperas post cesareadas en el Hospital Regional Docente de Trujillo [Tesis para obtener maestría en salud pública]. Perú, Universidad Nacional de Trujillo; 2015.
12. Mamani C. Factores de riesgo asociados a infección de sitio operatorio en postcesareadas del Hospital Carlos Monge Medrano, Juliaca 2016-2017. [Tesis]. Puno, Perú. Universidad Nacional del Altiplano; 2018.

13. Miranda S. Factores de riesgo asociados a infección de herida post cesárea, Hospital Regional de Occidente, 2010. [Tesis para obtener el grado de Magíster]. Guatemala. Universidad De San Carlos De Guatemala; 2013.

\section{PANACEA

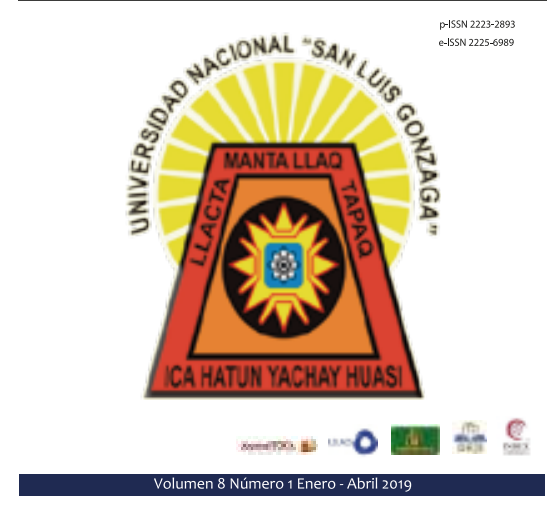

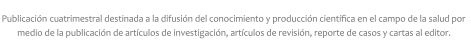

